\title{
Influence of stochastic magnetic fields on relativistic electrons
}

\author{
A. Wingen ${ }^{1}$, S.S. Abdullaev ${ }^{2}$, K.H. Finken ${ }^{2}$, M. Jakubowski ${ }^{2}$ and \\ K.H. Spatschek ${ }^{1}$ \\ ${ }^{1}$ Institut für Theoretische Physik, Heinrich-Heine-Universität Düsseldorf, D-40225 \\ Düsseldorf, Germany \\ ${ }^{2}$ Institut für Plasmaphysik, Forschungszentrum Jülich GmbH, EURATOM Association, \\ Trilateral Euregio Cluster, D-52425 Jülich, Germany \\ E-mail: spatschek@thphy.uni-duesseldorf.de
}

Received 5 May 2006, accepted for publication 25 September 2006

Published 18 October 2006

Online at stacks.iop.org/NF/46/941

\begin{abstract}
The relativistic motion of test particles in stochastic magnetic fields is investigated. Guiding-centre motion is analysed in relativistic invariant form for toroidal geometry. Including stochastic magnetic field components, a symmetric Hamiltonian mapping technique, leading to a 4-dimensional iteration procedure, is developed. In general, an external electric field and a time-dependence of the magnetic field perturbations are allowed for. Break-up of drift surfaces is demonstrated via Poincaré plots. The latter are analysed in detail for increasing (relativistic) kinetic energies of the particles. The dependence of the escape rates on the kinetic energy is calculated and compared with the escape rates for field lines. The non-relativistic limit of the model is derived. Quantitative results for the magnetic perturbations in a dynamic ergodic divertor of the TEXTOR experiment are shown, and predictions for runaway electrons are compared with experiments.
\end{abstract}

PACS numbers: 05.45.Ac, 45.20.Jj, 45.50.Dd, 52.20.Dq, 52.55.Dy, 52.55.Fa,52.55.Rk

\section{Introduction}

Typically, the transport rates of hot and magnetically confined plasmas deviate significantly from classical [1] or neoclassical [2] predictions. There exist many attempts to create a selfconsistent theory of nonlinear transport (see, e.g. [3,4] and references therein). Here, we further develop the idea of analysing test particle transport in the presence of given (magnetic) fluctuations [5]. This type of research is primarily justified by its contribution to the understanding of fundamental processes in plasmas [4]. In addition, recently test particle transport in stochastic magnetic fields received increasing practical interest because of new experiments with externally generated ergodic magnetic fields. It has been shown that, e.g. edge localized modes [6], plasma rotation [7] as well as the topology of heat flow patterns [8] at the boundary of stochastic edge plasmas are strongly influenced by the stochastic magnetic field line structures.

The magnetic field line dynamics is one aspect [9]. As is well known, magnetic field lines represent a $1+\frac{1}{2}$-degreesof-freedom Hamiltonian system. This fact is important for the description of field lines by flux-preserving mappings which are computationally efficient and powerful tools to study field lines in the presence of non-axisymmetric magnetic perturbations. Numerous mapping models of field lines in a toroidal system have been proposed to study the destruction of nested magnetic surfaces and the formation of stochastic magnetic field lines (see [10-17] and references therein).

Another aspect of the transport theory is the dynamics of particles in a magnetic field. It is known that in inhomogeneous magnetic fields particle orbits deviate from the magnetic field lines [18]. An important question is how this can affect the transport of particles in a stochastic magnetic field. The enhanced transport of heat and particles due to destroyed nested magnetic surfaces has been analysed in the past in a number of publications (see [19-26]).

In this paper, we investigate the drift motion of particles in stochastic magnetic fields. Specifically, we consider the dynamics of relativistic particles (runaway electrons) in the TEXTOR tokamak in the presence of the dynamic ergodic divertor (DED) $[16,27]$. The study of runaway electrons is important for magnetic confinement and interpretation of astrophysical observations [28-37]. The runaway phenomenon results from the strong increase in the mean free path of an electron with its velocity. When electrons, moving in an electric field, exceed a critical velocity, they are accelerated freely and can reach very high energies. Several mechanisms for runaway generation have been proposed [38]. Due to their synchrotron radiation, runaways lead to energy loss. The typical energy scale of particles in a fusion device 
ranges from a few $\mathrm{keV}$ up to $15 \mathrm{MeV}$ and more. In large tokamaks runaway electrons with energies up to several tens of $\mathrm{MeV}$ hav been observed during numerous disruptions (see [39] and references therein). In a tokamak, runaways can cause severe damage since they are deposited very locally at the first wall components $[40,41]$.

Avoidance, suppression and termination of runaway electrons in tokamaks became important issues for the future fusion devices. Experiments in JT-60U [42, 43] have demonstrated that the magnetic stochasticity may suppress runaway electrons. Runaway losses due to stochastic magnetic field perturbations have also been observed in Tore Supra during the ergodic divertor operation [44] and in the TEXT with the ergodic limiter $[45,46]$. Thus, it is very important to study how runaway orbits will be influenced by stochastic fields [23, 24, 45, 47- 55].

In a weakly collisional plasma, charged particles gyrate around the magnetic field lines. Since the gyro-radius scales with the magnetic field strength, for magnetic fields of the order of tesla the gyro-radii (especially for electrons) are very small compared with the dimensions of the system. Because of the curvature and gradients of the magnetic field, the particles drift away from the magnetic surfaces. In the guiding-centre approximation, one neglects finite gyro-radius effects. For integrable situations, the deviations of the drift surfaces from the magnetic KAM surfaces increase with the particle energy. The kinetic energy of the particles is therefore, in addition to the perturbation current for the stochastic magnetic field generation, an important parameter for the break-up of drift surfaces.

To study runaway electrons, e.g. with applications to the TEXTOR-DED, we generalize the non-relativistic Hamiltonian equations for guiding-centre motion, recently proposed in [56, 57], to the relativistic regime. The Hamiltonian system is studied by reducing it to a symplectic mapping. The latter is constructed from the continuous system using the method of canonical transformation $[15,17,58$, 59]. The fast-running mapping procedure is constructed in a symplectic (volume-preserving) form, in contrast to numerical mapping schemes for toroidal systems used in $[24,26,60]$. Other relativistic theories of guiding-centre motion in a tokamak plasma were also proposed in $[23,51$, 61,62]. Particularly, in [62] a specific mapping describing the dynamics of runaway electrons has been obtained.

The relativistic model derived here includes, besides stochastic magnetic fields, particle drift effects in toroidal geometry. In its general formulation, it describes particles of any charge, mass, kinetic energy and relative direction of motion. The general formulation of the theory also includes an arbitrary external electric field. Note that the particles are still described as test particles, meaning that the electric and magnetic fields are not self-consistent. Any influences of the particles on the fields are neglected. Furthermore, the model does not include collisions and trapping of particles.

The paper is organized as follows. In section 2 we formulate the relativistic drift Hamiltonian and derive the equations of motion for the guiding-centres in external electromagnetic (EM) fields. In section 3 we outline the construction of a 4-dimensional mapping procedure for the guiding-centre drift Hamiltonian. For quantitative predictions, we use the TEXTOR-DED magnetic field configuration [57]. The 4-dimensional mapping reduces to a 2-dimensional description for static magnetic field perturbations. In the latter case, in section 4 , we explicitly investigate the changes in the drift surfaces with increasing kinetic energy for runaway electrons. Including magnetic perturbations, the changes in the Poincare sections with increasing kinetic energy are discussed. Furthermore, the dependence of the runaway escape rates on the kinetic energy and on the perturbation current is investigated. The theoretical results are compared with recent measurements at TEXTOR. The paper is concluded by a short discussion and outlook. Appendix A shows the different behaviour of co-passing electrons in stochastic fields. The non-relativistic limit is obtained in appendix B.

\section{Relativistic drift Hamiltonian}

\subsection{Charged relativistic particles in an EM field}

Let us consider a particle with mass $m_{0}$ and charge $q=$ $Z_{q} e\left(Z_{q}=-1\right.$ for electrons), where $e$ is the elementary charge. The EM field is described by the magnetic vector potential $\vec{A}$ and the electric scalar potential $\hat{\phi}$. The vector potential includes the magnetic equilibrium field as well as the perturbation field. The external electric field is $\vec{E}=$ $-\nabla \hat{\phi}$. We use cylindrical coordinates $(\hat{R}, \varphi, \hat{Z})$ for the toroidal geometry. $\varphi$ represents the toroidal angle of the torus, while $\hat{R}$ corresponds to the major radius. Using these coordinates, the relativistic particle Hamiltonian $\hat{H}$ can be formulated in a standard form. Due to the gauge invariance of the vector potential, we can assume $A_{\hat{R}}=0$, i.e. $\vec{A}=$ $\left(0, A_{\varphi}, A_{\hat{Z}}\right)$. The toroidal field is fully determined by the $A_{z}$ component of the vector potential. The poloidal field and the perturbation field are described by $A_{\varphi}$-component. External resonant magnetic perturbations may also contribute to the $A_{z}$ component. However, as shown in [13], in the case of the TEXTOR-DED these contributions are small in comparision to the $A_{\varphi}$ component, and they can be neglected.

We normalize the coordinates as follows:

$$
\begin{aligned}
& x=\frac{\hat{R}-R_{0}}{R_{0}}, \quad z=\frac{\hat{Z}}{R_{0}}, \quad t=\omega_{c} \hat{T}, \\
& p_{x, z}=\frac{\hat{p}_{r, z}}{m_{0} \omega_{c} R_{0}}, \quad p_{\varphi}=\frac{\hat{p}_{\varphi}}{m_{0} \omega_{c} R_{0}^{2}} .
\end{aligned}
$$

Here, $R_{0}$ is the major radius of the torus. The coordinates $x$ and $z$ represent a Cartesian coordinate system, perpendicular to the toroidal angle coordinate, with its centre at the geometrical centre of the torus tube. To normalize the time we use the gyro-frequency $\omega_{c}=e B_{0} / m_{0} c$, with the main magnetic field $B_{0}$. With the definitions

$$
\tilde{H}=\frac{\hat{H}}{m_{0} \omega_{c}^{2} R_{0}^{2}}, \quad \phi=\frac{q}{m_{0} \omega_{c}^{2} R_{0}^{2}} \hat{\phi},
$$

we obtain

$$
\tilde{H}=\left[\varepsilon_{0}^{2}+\varepsilon_{0}\left(p_{x}^{2}+\frac{\left(p_{\varphi}-f_{\varphi}\right)^{2}}{(1+x)^{2}}+\left(p_{z}-f_{z}\right)^{2}\right)\right]^{1 / 2}+\phi
$$


where $\varepsilon_{0}=c^{2} / \omega_{c}^{2} R_{0}^{2}$ is the normalized particle rest energy and the normalized vector potential has the components

$$
f_{\varphi}=\frac{Z_{q}}{B_{0} R_{0}}(1+x) A_{\varphi}, \quad f_{z}=\frac{Z_{q}}{B_{0} R_{0}} A_{\hat{Z}} .
$$

It is convenient to expand the Hamiltonian to the 8-dimensional phase space $\left(q_{i}, p_{i}\right)=\left(x, \varphi, z, t, p_{x}, p_{\varphi}, p_{z}, p_{t}\right), i=$ $1, \ldots, 4$, including the time $t$ and the energy $\tilde{H}$ as additional canonical variables. The reason is that for the mapping technique we will have to change the independent variable to the toroidal angle $\varphi$. Defining the canonical momentum, conjugate to the time $t$, as $p_{t}=-\tilde{H}$, we can introduce

$U=\frac{1}{2}\left(\frac{\left(p_{\varphi}-f_{\varphi}\right)^{2}}{(1+x)^{2}}+\left(p_{z}-f_{z}\right)^{2}-\frac{\left(-p_{t}-\phi\right)^{2}}{\varepsilon_{0}}+\varepsilon_{0}\right)$

as an effective potential for a one-dimensional particle motion. In the following we will use the Hamiltonian

$$
\begin{aligned}
H= & \frac{1}{2}\left(p_{x}^{2}+\frac{\left(p_{\varphi}-f_{\varphi}\right)^{2}}{(1+x)^{2}}+\left(p_{z}-f_{z}\right)^{2}\right. \\
& \left.-\frac{\left(-p_{t}-\phi\right)^{2}}{\varepsilon_{0}}+\varepsilon_{0}\right)=\frac{1}{2} p_{x}^{2}+U=0 .
\end{aligned}
$$

The particle dynamics, including the gyration, follows from the canonical equations of motion

$$
\frac{\mathrm{d} q_{i}}{\mathrm{~d} \tau}=\frac{\partial H}{\partial p_{i}}, \quad \frac{\mathrm{d} p_{i}}{\mathrm{~d} \tau}=-\frac{\partial H}{\partial q_{i}}
$$

with the time-like parameter $\tau$. The latter is the relativistic reference time within the co-moving frame of reference.

\subsection{Guiding-centre approximation}

To simplify the problem, we transform the fast varying variables $\left(x, \varphi, z, t, p_{x}, p_{\varphi}, p_{z}, p_{t}\right)$ to the slowly-varying guiding-centre coordinates $\left(\vartheta_{x}, \Phi, Z, T, I_{x}, P_{\varphi}, P_{z}, P_{t}\right)$ to eliminate the fast gyration. The transformation is generated by the function

$$
F=\varphi P_{\varphi}+z P_{z}+t P_{t}+\varepsilon S\left(x, I_{x}, \varphi, P_{\varphi}, z, P_{z}, t, P_{t}\right),
$$

which depends on old coordinates and new momenta. Here,

$$
\varepsilon S=\int p_{x}\left(x^{\prime}, I_{x}, \varphi, P_{\varphi}, z, P_{z}, t, P_{t}\right) \mathrm{d} x^{\prime},
$$

and $\varepsilon$ is a small parameter given by the ratio $\rho_{x} / L$ of the gyro-radius $\rho_{x}$ to the characteristic scale $\mathrm{L}$ of the system. We use (10) to transform the radial coordinates $x, p_{x}$ to the actionangle variables $\vartheta_{x}, I_{x}$ via

$$
I_{x}=\frac{1}{2 \pi} \oint_{C} p_{x}(x) \mathrm{d} x, \quad \vartheta_{x}=\varepsilon \frac{\partial S}{\partial I_{x}} .
$$

The integration is along one full-turn gyration $C$ of the particle in the $\left(x, p_{x}\right)$-plane.

The guiding-centre is determined by the minimum of the effective potential $U(x)$. Due to the smallness of the gyro-radius, we Taylor-expand around the guiding-centre position $x_{c}$ :

$$
H=\frac{1}{2} p_{x}^{2}+U\left(x_{c}\right)+\frac{1}{2} \omega_{x}^{2}\left(x-x_{c}\right)^{2}+\mathcal{O}\left(\left(x-x_{c}\right)^{3}\right) .
$$

The condition for the minimum leads to

$$
u_{\varphi}^{2}+u_{\varphi} \frac{\partial f_{\varphi}}{\partial x}+(1+x) u_{z} \frac{\partial f_{z}}{\partial x}-(1+x) \gamma \frac{\partial \phi}{\partial x}=0
$$

with

$$
u_{\varphi}=\frac{p_{\varphi}-f_{\varphi}}{1+x}, \quad u_{z}=p_{z}-f_{z}, \quad \gamma=\frac{-p_{t}-\phi}{\varepsilon_{0}} .
$$

The solution $x_{c}=x_{c}\left(\varphi, z, t, p_{\varphi}, p_{z}, p_{t}\right)$ of equation (13) is the position of the guiding-centre. The second derivative at $x_{c}$ defines the gyro-frequency

$$
\omega_{x}\left(x_{c}, \Phi, Z, T, I_{x}, P_{\varphi}, P_{z}, P_{t}\right)=\left|\frac{\partial^{2} U}{\partial x^{2}}\right|_{x=x_{c}}^{1 / 2} .
$$

Next, we transform making use of (9) and (10). We expand the Hamiltonian into a power series in $\varepsilon$ and obtain

$$
\begin{aligned}
H & =H_{0}+\varepsilon H_{1}\left(\vartheta_{x}, \Phi, Z, T, I_{x}, P_{\varphi}, P_{z}, P_{t}\right)+O\left(\varepsilon^{2}\right) \\
& =\omega_{x}\left(x_{c}, \Phi, Z, T, I_{x}, P_{\varphi}, P_{z}, P_{t}\right) I_{x} \\
& +U\left(x_{c}, \Phi, Z, T, I_{x}, P_{\varphi}, P_{z}, P_{t}\right) \\
& +\varepsilon H_{1}+O\left(\varepsilon^{2}\right)=0 .
\end{aligned}
$$

The zeroth-order in $\varepsilon$ describes the guiding-centre motion. It does not depend on the fast gyro-phase $\vartheta_{x}$. As shown in [56], the action variable $I_{x}$ is a good adiabatic invariant for typical tokamak plasmas. Also, the gyro-frequency $\omega_{x}$ is much larger than the toroidal and poloidal transit frequencies of the drift motion. Therefore, we can neglect the first and higher orders in $\varepsilon$ for electrons.

For the relations between the geometrical and guidingcentre coordinates, we have to determine the unknown part $S$ of the generating function. For this we solve equation (12) with respect to $p_{x}$ :

$$
p_{x}=\sqrt{2\left(H-U\left(x_{c}\right)\right)-\omega_{x}^{2}\left(x-x_{c}\right)^{2}},
$$

and get from equation (10)

$$
\varepsilon S=\int \sqrt{2 I_{x} \omega_{x}-\omega_{x}^{2}\left(x^{\prime}-x_{c}\right)^{2}} \mathrm{~d} x^{\prime} .
$$

Substituting

$$
y=\frac{x^{\prime}-x_{c}}{\sqrt{2 I_{x} / \omega_{x}}},
$$

we find

$$
\varepsilon S=2 I_{x} \int \sqrt{1-y^{2}} \mathrm{~d} y=I_{x}\left[\arcsin y+y \sqrt{1-y^{2}}\right] .
$$

The angle variable $\vartheta_{x}$, conjugate to the action-variable $I_{x}$, reads

$$
\vartheta_{x}=\frac{\partial S}{\partial I_{x}}=\arcsin y
$$

The relations between the old coordinates $\left(q_{i}, p_{i}\right)$ and the guiding-centre coordinates $\left(Q_{i}, P_{i}\right)$ are given by

$$
Q_{i}=\frac{\partial F}{\partial P_{i}}, \quad p_{i}=\frac{\partial F}{\partial q_{i}}, \quad i=1, \ldots, 4 .
$$

The action variable $I_{x}$ is considered a constant of motion. 


\subsection{Simplification of the guiding-centre equations}

We can simplify the equations for the guiding-centres and the relations between the particle and guiding-centre coordinates significantly by neglecting perturbations in comparison to the main toroidal magnetic field $B_{\varphi}=B_{0} R_{0} / \hat{R}$. This leads to the ansatz

$A_{z}(\hat{R})=-B_{0} R_{0} \ln \frac{\hat{R}}{R_{0}}, \quad$ i.e. $f_{z}=-Z_{q} \ln (1+x)$

for the $z$ component of the vector potential. We also can neglect the terms in equation (13) being proportional to $u_{\varphi}^{2}, u_{\varphi} \frac{\partial f_{\varphi}}{\partial x}$ and $\frac{\partial \phi}{\partial x}$. For relativistic electrons we typically have $u_{\varphi} \approx 10^{-2}$, while all field components are small compared to $B_{0}$ [56]. Then we obtain from equation (13)

$$
(1+x)\left(p_{z}-f_{z}\right) \frac{\partial f_{z}}{\partial x}=0, \quad \text { i.e. } p_{z}=f_{z}
$$

and

$$
x_{c}=\mathrm{e}^{-p_{z} / Z_{q}}-1 .
$$

According to equation (15)

$$
\omega_{x}=\frac{1}{1+x_{c}}=\mathrm{e}^{p_{z} / Z_{q}} .
$$

The relations between the particle and guiding-centre coordinates simplify to

$$
\begin{array}{ll}
x=x_{c}+\sqrt{\frac{2 I_{x}}{\omega_{x}}} \sin \vartheta_{x}, \quad p_{x}=\sqrt{2 I_{x} \omega_{x}} \cos \vartheta_{x}, \\
z=Z+\sqrt{\frac{2 I_{x}}{\omega_{x}}} \cos \vartheta_{x}, \quad p_{z}=P_{z}, \\
\varphi=\Phi, \quad p_{\varphi}=P_{\varphi}, \quad t=T, \quad p_{t}=P_{t} .
\end{array}
$$

Neglecting the fast gyro-phase, the Hamiltonian (16) reads

$$
\begin{gathered}
H=\omega_{x}\left(p_{z}\right) I_{x}+\frac{1}{2}\left[\frac{\left(p_{\varphi}-f_{\varphi}\left(x_{c}, \varphi, z, t\right)\right)^{2}}{\left(1+x_{c}\right)^{2}}\right. \\
\left.-\frac{\left(-p_{t}-\phi\left(x_{c}, \varphi, z, t\right)\right)^{2}}{\varepsilon_{0}}+\varepsilon_{0}\right]=0 .
\end{gathered}
$$

The particle dynamics is given by the Hamiltonian equations of motion (8) with the coordinates $q_{i}=\left(\vartheta_{x}, z, \varphi, t\right)$ and the canonical momenta $p_{i}=\left(I_{x}, p_{z}, p_{\varphi}, p_{t}\right)$.

\subsection{Hamiltonian equations for passing particles}

For passing particles, the Hamiltonian drift equations can be reformulated by introducing the toroidal angle $\varphi$ as the independent, time-like variable and the corresponding canonical momentum $p_{\varphi}$ as a new Hamiltonian $K=-p_{\varphi}$ (see [56]). From equation (30) we find

$$
\omega_{x} I_{x}+\frac{1}{2}\left[\varepsilon_{0}-\frac{\left(-p_{t}-\phi\right)^{2}}{\varepsilon_{0}}\right]=-\frac{1}{2} \frac{\left(-K-f_{\varphi}\right)^{2}}{\left(1+x_{c}\right)^{2}}
$$

i.e.

$$
K=-f_{\varphi}-\sigma\left(1+x_{c}\right)\left[\varepsilon_{0}\left(\gamma^{2}-1\right)-2 \omega_{x} I_{x}\right]^{1 / 2} .
$$

Here we introduce the new parameter $\sigma=u_{\varphi} /\left|u_{\varphi}\right|= \pm 1$, which determines the direction of motion relative to the field lines. For $\sigma=1$ the particles move in the positive direction of the toroidal angle (co-passing particles). For $\sigma=-1$ the particles move in the opposite direction, the so-called counter-passing particles. The dynamics is given by the Hamiltonian equations of motion, now with respect to the new Hamiltonian $K$,

$$
\begin{aligned}
\frac{\mathrm{d} z}{\mathrm{~d} \varphi} & =\frac{1}{Z_{q}}\left(1+x_{c}\right)\left[\frac{\partial f_{\varphi}}{\partial x_{c}}\right. \\
& \left.+\sigma \frac{\varepsilon_{0}\left(\gamma^{2}-1\right)-\omega_{x} I_{x}-\left(1+x_{c}\right) \gamma \frac{\partial \phi}{\partial x_{c}}}{\sqrt{\varepsilon_{0}\left(\gamma^{2}-1\right)-2 \omega_{x} I_{x}}}\right],
\end{aligned}
$$

$\frac{\mathrm{d} p_{z}}{\mathrm{~d} \varphi}=\frac{\partial f_{\varphi}}{\partial z}-\sigma \frac{\partial \phi}{\partial z} \frac{\left(1+x_{c}\right) \gamma}{\sqrt{\varepsilon_{0}\left(\gamma^{2}-1\right)-2 \omega_{x} I_{x}}}$,

$\frac{\mathrm{d} t}{\mathrm{~d} \varphi}=\sigma \frac{\left(1+x_{c}\right) \gamma}{\sqrt{\varepsilon_{0}\left(\gamma^{2}-1\right)-2 \omega_{x} I_{x}}}$

$\frac{\mathrm{d} p_{t}}{\mathrm{~d} \varphi}=\frac{\partial f_{\varphi}}{\partial t}-\sigma \frac{\partial \phi}{\partial t} \frac{\left(1+x_{c}\right) \gamma}{\sqrt{\varepsilon_{0}\left(\gamma^{2}-1\right)-2 \omega_{x} I_{x}}}$,

$\frac{\mathrm{d} \vartheta_{x}}{\mathrm{~d} \varphi}=\sigma\left(\varepsilon_{0}\left(\gamma^{2}-1\right)-2 \omega_{x} I_{x}\right)^{-1 / 2}$,

$\frac{\mathrm{d} I_{x}}{\mathrm{~d} \varphi}=0$.

For the co-passing particles $(\sigma=+1)$ the set of Hamiltonian equations (33)-(38) should be integrated along a positive direction of the toroidal angle $\varphi$, and vice versa for counter-passing particles $(\sigma=-1)$.

\section{Discrete mapping}

\subsection{Specification of a magnetic field configuration}

The toroidal component $A_{\varphi}$ of the vector potential is modelled according to [57]. The unperturbed part is given by

$$
A_{\varphi}^{(0)}=\frac{B_{0} R_{0}}{1+x} \int \frac{\mathrm{d} \psi}{q(\rho(\psi))}, \quad \text { i.e. } f_{\varphi}^{(0)}=Z_{q} \int \frac{\mathrm{d} \psi}{q(\rho(\psi))} .
$$

Here, $q(\rho)$ is the safety factor

$$
\begin{aligned}
& q(\rho)=q_{a} \frac{\rho^{2} / a^{2}}{1-\left(1-\frac{\rho^{2}}{a^{2}}\right)^{v}} \quad \text { for } \rho \leqslant a, \\
& q(\rho)=q_{a} \frac{\rho^{2}}{a^{2}} \quad \text { for } \rho>a .
\end{aligned}
$$

$q_{a}=2 \pi B_{0} R_{0} a^{2} \mu_{0}^{-1} I_{p}^{-1}$ is the safety factor at the plasma edge $a$, and the exponent $v=q_{a} / q_{0} . \quad I_{\mathrm{p}}$ is the plasma current. $\psi=\frac{1}{2} \rho^{2}$ is the toroidal magnetic flux (action variable), and $\rho$ is the minor radius of the magnetic KAM surfaces. We have a Shafranov shift $[16,57,63]$

$$
\Delta(\rho)=R(\rho)-R_{a} \approx \eta\left(a^{2}-\rho^{2}\right)
$$

with a given parameter $\eta$. The relation between the minor radius $\rho$ and the geometrical coordinates reads

$$
\rho=\sqrt{\left(1+x-R_{a}-\Delta(\rho)\right)^{2}+z^{2}} .
$$


$R_{a}$ is the major radius of the plasma centre. Since the Shafranov shift itself depends on the shifted radius $\rho$, equation (43) is an implicit equation. It can be solved for $\rho$.

According to [16], for the TEXTOR-DED the normalized vector potential of the perturbation magnetic field is approximated as

$$
f_{\varphi}^{(1)}(x, z, \varphi, t)=\epsilon \sum_{m} f_{m}(\bar{r}, \theta) \cos (m \theta+n \varphi+\omega t)
$$

with the Fourier modes

$$
\begin{aligned}
f_{m}(\bar{r}, \theta) & =-\bar{r}_{c} g_{m} m^{-1} \sqrt{1+\bar{r} \cos \theta}\left(\frac{\bar{r}}{\bar{r}_{c}}\right)^{m}, \\
g_{m} & =(-1)^{m} \frac{\sin \left[\left(m+n m_{0} / 4\right) \theta_{c}\right]}{\left(m+n m_{0} / 4\right) \pi} .
\end{aligned}
$$

Here $\epsilon=B_{c} / B_{0}$ stands for the perturbation parameter, $\bar{r}=$ $\sqrt{x^{2}+z^{2}}$ is the normalized minor radius of the torus and $\theta=\arctan (z / x)$ is the poloidal angle, $\bar{r}_{c}=r_{c} / R_{0}$ where $r_{c}$ is a minor radius of the DED-coils. The dimensional minor radius $r$ is $r=\bar{r} R_{0}$.

There are three operational modes of the DED: $m / n=$ $12 / 4,6 / 2$ and $3 / 1$. In these modes the toroidal mode number $n$ takes the value $n=4$ for the $12 / 4$ mode, $n=2$ for the $6 / 2$ mode and $n=1$ for the $3 / 1$ mode, respectively.

\subsection{Details of the relativistic drift map}

Presenting the normalized vector potential $f$ as a sum, $f=$ $f_{\varphi}^{(0)}(x, z)+f_{\varphi}^{(1)}(x, z, \varphi, t)$, we write the Hamiltonian (32) in the form

$$
K=K_{0}\left(z, p_{z}, p_{t}\right)+K_{1}\left(z, t, p_{z}, p_{t}, \varphi\right),
$$

where $K_{0}\left(z, p_{z}, p_{t}\right)$ is the unperturbed Hamiltonian defined by equation (32) with $f=f_{\varphi}^{(0)}(x, z)$, and $K_{1}=-f_{\varphi}^{(1)}$ describes the perturbation Hamiltonian.

The construction scheme of a symplectic mapping for the system (45) is given in $[15,17,57,59]$ and consists of the following steps. First, for the unperturbed Hamiltonian $K_{0}\left(z, p_{z}, p_{t}\right)$, one introduces the action-angle variables $(I, \vartheta)$ for the canonical variables, $\left(z, p_{z}\right)$. In these variables the unperturbed Hamiltonian $K_{0}$ depends only on the action variable $I$ and the energy $p_{t}$, i.e. $K_{0}=K_{0}\left(I, p_{t}\right)$. One introduces also a 'time' variable $T$ being conjugate to the energy $p_{t}$. The perturbation Hamiltonian $K_{1}=$ $K_{1}\left(I, \vartheta, p_{t}, T\right)$ is a periodic function of the angle $\vartheta$ with a period $2 \pi$, and can be presented as a Fourier series in $\vartheta$. The corresponding Fourier coefficients $K_{m}\left(I, p_{t}\right)$ are also precalculated.

For this Hamiltonian the drift orbits are $\boldsymbol{q}(\varphi)=$ $\left(I(\varphi), \vartheta(\varphi), p_{t}(\varphi), T(\varphi)\right)$. The forward and backward mappings are defined as

$$
\left(I_{k \pm 1}, \vartheta_{k \pm 1}, p_{k \pm 1}, T_{k \pm 1}\right)=\hat{T}_{ \pm}\left(I_{k}, \vartheta_{k}, p_{k}, T_{k}\right),
$$

which relates the intersection points, $\left(I_{k}, \vartheta_{k}, p_{k}, T_{k}\right),(k=$ $0, \pm 1, \pm 2, \ldots)$, of the drift orbit $\boldsymbol{q}(\varphi)$ at the poloidal sections $\varphi_{k}$ and $\varphi_{k \pm 1}$. The poloidal sections can be defined as $\varphi_{k}=$ $k(2 \pi / s)$, where $s \geqslant 1$ is an integer number.

The explicit form of the 4D mapping (46) is given in [57]. For the time-independent perturbations $(\omega=0)$, the mapping

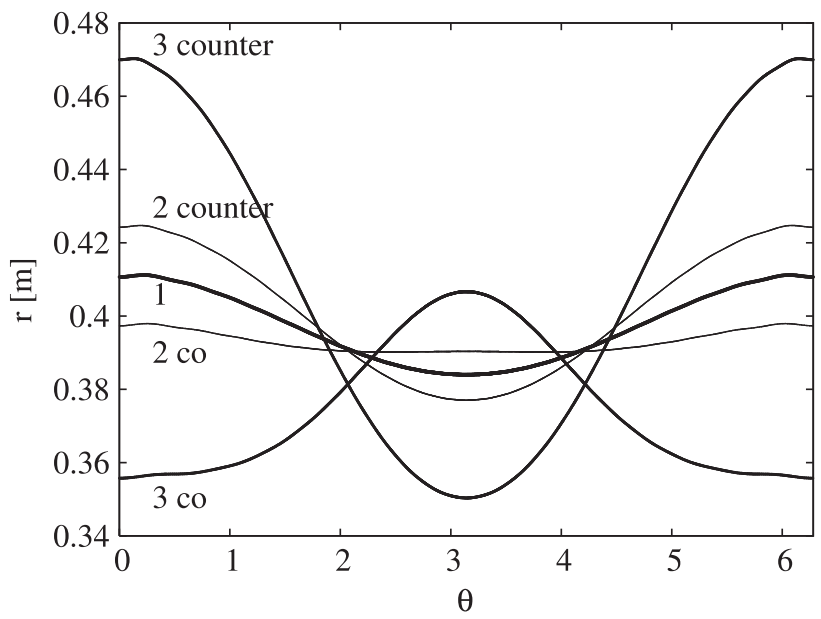

Figure 1. Unperturbed drift surfaces for various kinetic energies compared with the magnetic surface (label 1). Shown are surfaces for $q=\pi$, with kinetic energies of $2 \mathrm{MeV}$ (label 2) and $10 \mathrm{MeV}$ (label 3) for co- and counter-passing particles, respectively.

reduces to the two-dimensional case. Then the particle energy $p_{t}$ is a constant of motion. In the following we evaluate the two-dimensional case. The mappings $\hat{T}_{ \pm}$in this case read

$$
\begin{aligned}
& J_{k}=I_{k}-\varepsilon \frac{\partial S_{k}}{\partial \vartheta_{k}}, \quad \Theta_{k}=\vartheta_{k}+\varepsilon \frac{\partial S_{k}}{\partial J_{k}}, \\
& \bar{\Theta}_{k}=\Theta_{k}+\Omega\left(J_{k}\right)\left(\varphi_{k \pm 1}-\varphi_{k}\right), \\
& I_{k \pm 1}=J_{k}+\varepsilon \frac{\partial S_{k \pm 1}}{\partial \vartheta_{k \pm 1}}, \quad \vartheta_{k \pm 1}=\bar{\Theta}_{k}-\varepsilon \frac{\partial S_{k \pm 1}}{\partial J_{k}},
\end{aligned}
$$

where $\Omega(I)=\partial K_{0} / \partial I$ is the transit frequency. The generating functions $S_{k}$ and $S_{k \pm 1}$ are given in [15, 17,57].

In practical calculations we use the generating function in the first order of small perturbation parameter $\epsilon$. The accuracy of mapping in this case depends on the map step, $\Delta \varphi=\varphi_{k+1}-\varphi_{k}$. As was shown in $[17,59]$ for $\Delta \varphi$ comparable with a characteristic period of perturbation the mapping has the same accuracy as the symplectic integrator with integration steps two orders smaller.

Finally, we should note that the mapping $\hat{T}_{+}$should be applied for co-passing particles while $\hat{T}_{-}$should be used for counter-passing particles. Particularly, since runaway electrons are counter-passing particles their orbits are mapped by $\hat{T}_{-}$.

\subsection{Unperturbed drift surfaces}

First, we consider drift orbits in the absence of magnetic perturbations. In this case the Hamiltonian $K=-p_{\varphi}$ (32) is the integral of motion, i.e. $p_{\varphi}=$ const. Therefore, the drift orbits lie on the surfaces determined by equation $p_{\varphi}(x, z)=$ const. These surfaces do not coincide with the magnetic surfaces $f_{\varphi}(x, z)=$ const. As seen from equation (32) the shift of drift orbits from the magnetic surfaces depends not only on the kinetic energy of particles but also on the direction of motion $\sigma$ and its charge.

Figure 1 shows various drift surfaces for different kinetic energies and propagation directions in comparison to the magnetic KAM surface for the field lines. We have chosen the constant irrational value $q=\pi$. The magnetic surface is 
shifted outwards, compared to the geometrical centre of the torus. This is caused by the Shafranov shift which takes into account the effects of the toroidal geometry. Note that the poloidal angle $\theta=\pi$ marks the inner side of the torus, where the perturbation coils are mounted, while $\theta=0$ corresponds to the outer side. As one can see from figure 1, the drift surfaces for co-passing electrons are shifted inwards. The shift is larger for higher energies and always larger than the shift in the magnetic surface. The latter can be interpreted as the drift surface of particles with zero energy. The counterpassing electrons are shifted outwards, and again the shift is larger for higher energies. The Shafranov shift is compensated at an energy of about $2 \mathrm{MeV}$.

\section{Runaways in perturbed fields}

Runaway electrons $(\leqslant 40 \mathrm{MeV})$ propagate in tokamaks opposite to the direction of the plasma current. In TEXTOR the plasma current and the mean toroidal magnetic field have opposite directions, while in our model both have the same direction. We can apply the formulation of the equations of motion provided here by a change of the sign in the definition of the normalized vector potential $f_{z}$ in equation (23), i.e. by $f_{z} \rightarrow-f_{z}$. Then in equations (25), (26) and (33) one should make the change $Z_{p} \rightarrow-Z_{p}$.

Measurements at TEXTOR [37] show that in the $m / n=$ $3 / 1$ base mode operation of the DED a relative wide area of runaway electrons is depopulated. In the DED $m / n=$ $12 / 4$ base mode, this area is considerably smaller. For the understanding of this phenomenon we analyse the structures of the Poincaré sections in all base mode operations for various kinetic energies. Further, we consider $N_{0}$ test particles on a certain unperturbed drift surface in the ergodic region and determine the escape rates of these test particles in all three base modes for different kinetic energies. The perturbation current is thereby kept constant.

\subsection{Broken runaway drift surfaces}

4.1.1. $m / n=12 / 4$ base mode. The mapping procedure (47)-(49) provides us with the Poincaré plot, which will be called a drift map in the following. Using the same parameters as in figure 1, we now discuss drift maps for finite perturbation currents. We start with $I_{0}=10 \mathrm{kA}$ and the $m / n=12 / 4$ base mode and we vary the energy of the runaway electrons.

The general tendency is as follows. The structures created by runaway electrons with the kinetic energy up to $1 \mathrm{MeV}$ are very similar to the structures of the field lines. This means that low-energy particles mainly follow the field lines. However, high-energetic electrons behave more regularly. This can be seen from the drift map for runaway electrons which becomes more and more regular with increasing particle energy. Also the finger structure [57] is less pronounced as for field lines. Figures 2(a) and $(b)$ show the drift map for runaway electrons at $0.5 \mathrm{MeV}$ and $8 \mathrm{MeV}$, respectively. As seen from figure 2(b), at the kinetic energy $8 \mathrm{MeV}$ the finger structures present at $0.5 \mathrm{MeV}$ (see figure 2(a)) vanished and the drift motion became very regular. All particles are confined inside the plasma, because the last surface directly beneath the wall is an intact drift surface. No chaotic behaviour remains. This has two
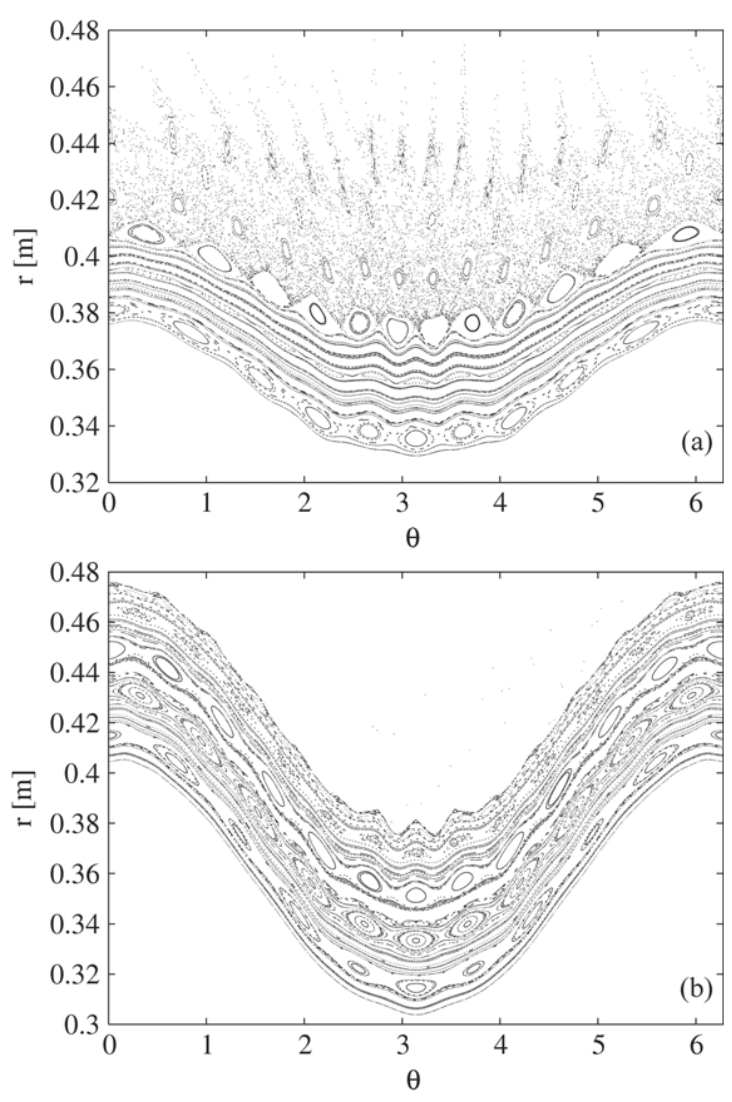

Figure 2. Drift maps for (a) $500 \mathrm{keV}$ and (b) $8 \mathrm{MeV}$ runaway electrons in the $m / n=12 / 4$ base mode at a perturbation current of $10 \mathrm{kA}$.

reasons. Due to the drift effects, the structures of runaway electrons are shifted outside, so that the particles are shifted away from the DED coils, as already shown in figure 1. They are shifted into areas of lower perturbations. The drift map becomes more and more regular for higher energies and also the vanishing of the finger structures [57] is expected. But this is not the only reason for the regular behaviour. Analysing the copassing electrons, one might expect a strong chaotic behaviour caused by the shift towards the coils. On the contrary, the runaway electrons behave more regular for higher energies too. This can only be attributed to the high kinetic energy of the electrons. Their motion inside the torus is so fast that they do not really feel the chaotic magnetic field.

We can conclude that generally particles with high kinetic energy do not really feel the chaotic magnetic field during the $m / n=12 / 4$ base mode. The high-energetic particles are confined inside the plasma, and they are strongly shifted to one side. The last intact drift surface is located directly beneath the wall, either at the outside, $\theta=0$, or at the inside, $\theta=\pi$, of the torus, depending on the particle charge and the direction of motion. Particles beyond the last intact drift surface are connected to the wall. They move on surfaces which cross the wall. These particles may be lost to the wall extremely fast. The drift maps for the $m / n=12 / 4$ base mode operation indicate that runaways are not affected by the stochastic magnetic fields.

We should note that similar results were also obtained in [23, 24, 47-49]. 


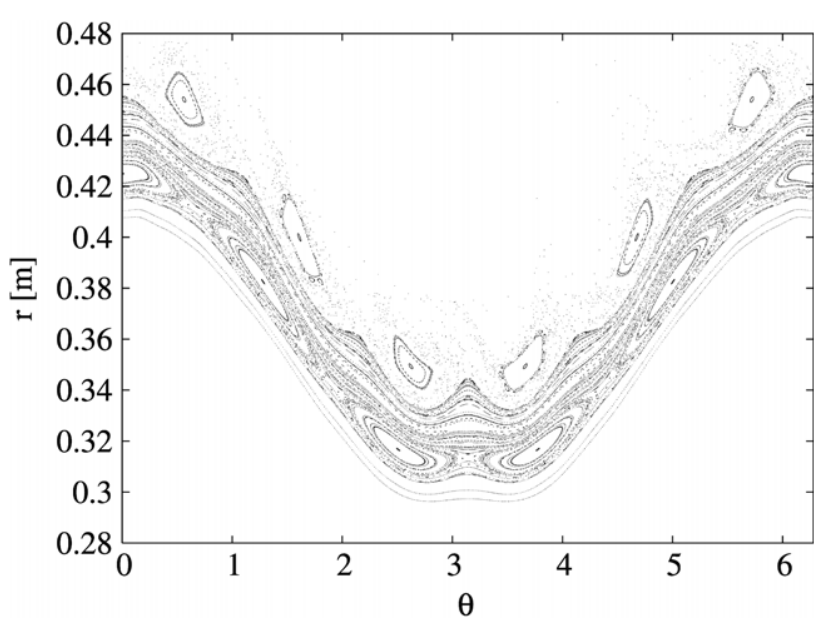

Figure 3. Drift map for $10 \mathrm{MeV}$ runaway electrons in the $m / n=6 / 2$ base mode at a perturbation current of $5 \mathrm{kA}$.

In appendix A we present results for co-passing electrons in the $12 / 4$ mode operation.

4.1.2. $m / n=6 / 2$ base mode. The situation for the $m / n=$ $6 / 2$ mode is similar to the $m / n=12 / 4$ mode, although the islands are larger and the perturbation penetrates more deeply into the plasma edge compared with the $12 / 4$ mode operation.

Figure 3 shows the Poincaré section of $10 \mathrm{MeV}$ runaway electrons in the $6 / 2$ mode. Similar to the $12 / 4$ mode the high-energetic particles behave more regularly than the lowenergetic ones. In comparison to the $12 / 4$ mode the $10 \mathrm{MeV}$ particles are much more chaotic in $6 / 2$ mode operation than the $8 \mathrm{MeV}$ in $12 / 4$ mode. As one can see in figure 3 there are still areas of stochasticity and the system is still open to the wall, but one can also see that an intact drift surface has been formed at the transition of the ergodic zone to the laminar zone. At $10 \mathrm{MeV}$ the runaway electrons of the ergodic zone are confined in the plasma. In the $6 / 2$ mode the runaway particles are very well affected by the stochastic field. One can expect that the number of runaway electrons decreases with DED operation. Many high-energetic particles are lost at the wall before their kinetic energy has reached values where they are confined.

4.1.3. $m / n=3 / 1$ base mode. In the $3 / 1$ mode operation the perturbation field penetrates the plasma even deeper than in the $6 / 2$ mode.

In spite of the high kinetic energy of $15 \mathrm{MeV}$ figure 4 shows a very chaotic behaviour of the runaway electrons in the $3 / 1$ mode operation. The energy thresholds for forming stable drift surfaces is much larger in the $3 / 1$ mode than in the $6 / 2$ or $12 / 4$ mode. We can expect that the loss of runaway electrons is significantly large in the $3 / 1$ mode operation. The area affected by the perturbation and being open to the wall is larger than in the 6/2 mode, and also the high-energetic particles feel the perturbation.

\subsection{Escape rates}

In the absence of external magnetic perturbations the runaway electrons leave the plasma region mostly due to collisional diffusion. The rate of such a diffusion in TEXTOR is of

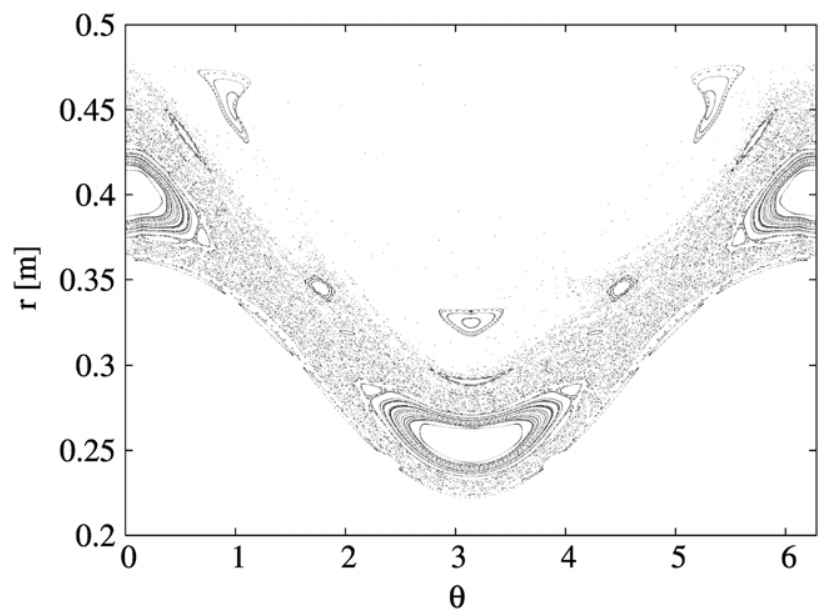

Figure 4. Drift map for $15 \mathrm{MeV}$ runaway electrons in the $m / n=3 / 1$ base mode at a perturbation current of $3 \mathrm{kA}$.

the order of $0.01 \mathrm{~m}^{2} \mathrm{~s}^{-1}$ [50]. In the presence of magnetic perturbations, the runaway diffusion coefficient $D_{r}$ for lowenergy electrons with a few $\mathrm{MeV}$ can be simply estimated using the formula $D_{r}=v_{r} D_{m}$, where $D_{m}$ is the diffusion coefficient of field lines and $v_{r}$ is a typical velocity of electrons. For the $m / n=3 / 1$ base mode $D_{m}$ is of the order of $10^{-5} \mathrm{~m}$ at the perturbation current $I_{0}=3.75 \mathrm{kA} \mathrm{[16]}$. Therefore, for electrons with $1 \mathrm{MeV}$ we have $D_{r} \approx 3 \times 10^{3} \mathrm{~m}^{2} \mathrm{~s}^{-1}$, which means that the confinement time is $\tau_{r} \approx \Delta r / \sqrt{2 D_{r}} \approx 1 \mu \mathrm{s}$ (in the stochastic layer of width $\Delta r \approx 8 \mathrm{~cm}$ ). However, this estimation of the confinement time is valid only for the lowenergy runaway electrons, $E \leqslant 1 \mathrm{MeV}$, when drift orbits of runaway electrons are close to magnetic field lines.

As has been shown above, with increasing kinetic energy the drift orbits become less stochastic, and one expects a decrease in the diffusion rate due to stochastic fields. Calculations performed in [64] show that the quasilinear diffusion coefficients are reduced due to the effect of drifts. The quasilinear estimations are valid, however, only for the highly developed chaotic motion, and they are not applicable for partially chaotic systems. As shown in [12-16], the quasilinear predictions of field line diffusion coefficients in the stochastic layer of the TEXTOR-DED exceed the ones obtained by direct integrations by a factor of order of 10 . Therefore, for a realistic estimation of confinement times of runaway electrons in the partially stochastic layer one should use direct calculations.

Below we study the escape rates of runaway electrons for different energies. This will provide us with an estimate of the energy thresholds for the particle confinement in the different modes. We start with $N_{0}$ test particles on an unpertubed drift surface, being equally distributed along the whole poloidal angle. Then we iterate until the particles hit the wall, where they are eliminated. The calculation is stopped when $90 \%$ of the particles are lost. $N(t)$ is the number of particles in the system at time $t$. Thereby $t$ corresponds directly to the number of toroidal rotations given by the number of iterations. $N(t) / N_{0}$ is called the escape rate.

Figure 5 shows the calculated logarithm of the escape rates for field lines, $1.25 \mathrm{MeV}$, and $1.75 \mathrm{MeV}$ runaway electrons, respectively. The perturbation current is kept constant at $I_{0}=10 \mathrm{kA}$ in the $m / n=12 / 4$ base mode. The escape rates 


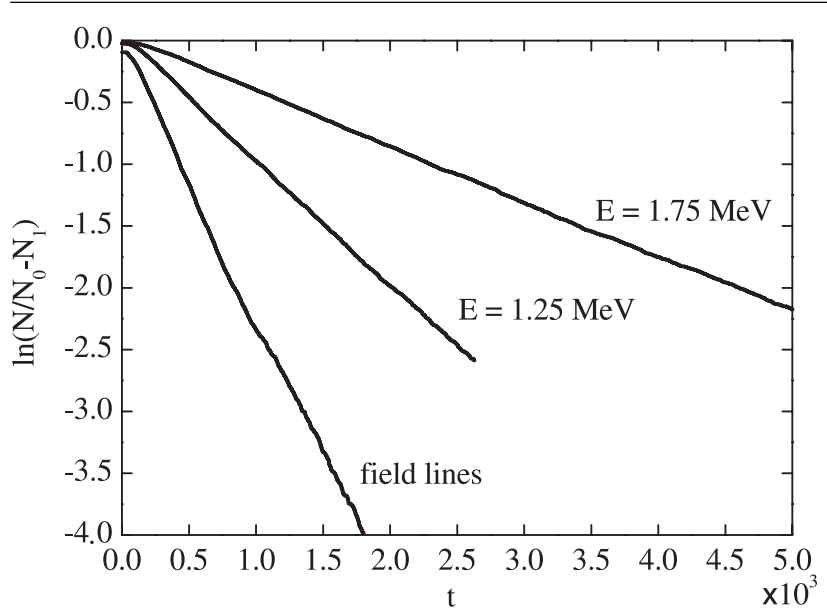

Figure 5. Logarithms of the escape rates for magnetic field lines and electrons (at two different energies), respectively, in the 12/4 mode.

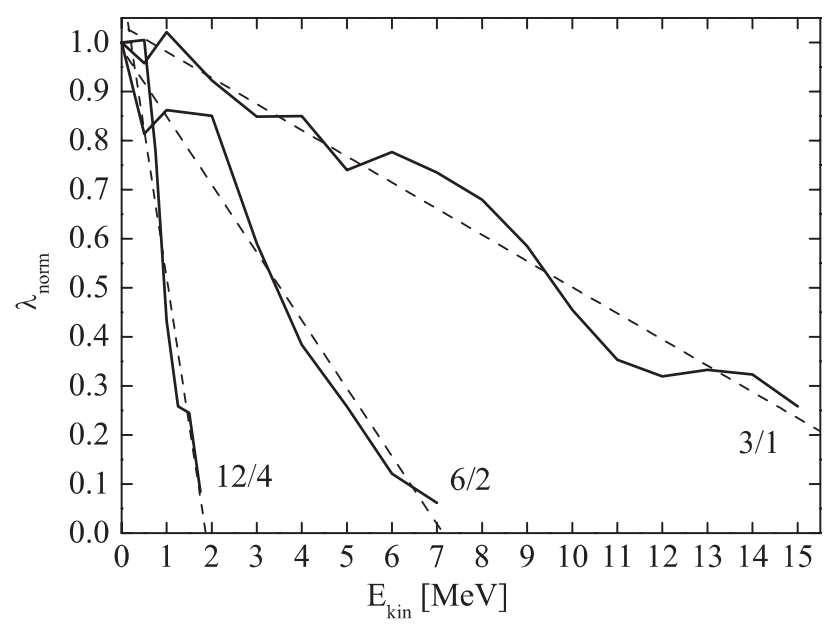

Figure 6. Exponential decay parameter $\lambda_{\text {norm }}$, normalized with the decay parameter for field lines. Shown is the dependence on the kinetic energy (solid lines; linear fits depicted as dashed lines) for different base mode operations.

follow an exponential decay law

$$
N(t)=N_{0}\left(N_{1}+\mathrm{e}^{-\lambda t}\right) .
$$

We can determine the decay parameter $\lambda$ from the gradient of the linear curves. Note that there is an offset $N_{1}$ of about $5-10 \%$ for all escape rates. The reason for this offset is unknown. One can speculate that extremely long running particles are either following another, very long time escape mechanism, or that they are not leaving the system at all. By calculating the escape rates for various kinetic energies and all three base mode operations, we find the dependence of the characteristic decay parameter $\lambda$ on the energy as

$$
\lambda \sim-\left(E-E_{c}\right) .
$$

Typical decay parameters are depicted in figure 6 for $I_{0}=10 \mathrm{kA}$ in the $m / n=12 / 4$ base mode, for $I_{0}=5 \mathrm{kA}$ in the $m / n=6 / 2$ base mode, and for $I_{0}=3 \mathrm{kA}$ in the $m / n=3 / 1$ base mode. The decay parameters decrease linearly with increasing energy. From figure 6 we can determine the critical energies $E_{c}$, where a stable drift surface is formed at the edge of the ergodic zone. Within our model (no collisions), for $E>E_{c}$ all particles from the ergodic zone are confined within the plasma. From $\lambda=0$ we obtain $E_{c, 12 / 4}=1.87 \mathrm{MeV}$ in the $m / n=12 / 4$ base mode. This explains why runaways are not significantly affected by the DED in the $m / n=12 / 4$ base mode operation. The laminar zone is still open to the wall. For higher kinetic energies more intact drift surfaces are formed beneath the wall. At $8 \mathrm{MeV}$ (see figure 2) the laminar zone is also confined. So, all high-energetic runaway electrons are confined inside the plasma, which agrees with experimental observations [37].

The critical energy $E_{c, 6 / 2}$ for establishing an intact drift surface at the edge of the ergodic region is much larger than for the $12 / 4$ mode. We obtain $E_{c, 6 / 2}=7.13 \mathrm{MeV}$, which is underlined by figure 3 , where the former ergodic region is already regular. The threshold for the total plasma confinement is expected to be much larger. Runaway electrons are much more affected by the stochastic field compared with the 12/4 mode operation. We expect an enormous decrease in runaway electrons with DED operation in the $6 / 2$ mode. In particular the lower-energetic runaways (a few $\mathrm{MeV}$ ) are lost at the wall while the very high-energetic ones are only slightly affected. This is confirmed by experiment as we will see later.

The extrapolation of the critical energy for the $3 / 1$ mode reads $E_{c, 3 / 1}=19.4 \mathrm{MeV}$. At this energy the ergodic zone is confined. Figure 4 shows that at $15 \mathrm{MeV}$ the ergodic zone is still open. We expect the energy threshold for the total confinement of the plasma to be much larger than the typical energy range of runaway electrons. In the $3 / 1$ mode operation all runaway electrons are affected strongly by the DED perturbation and we expect large losses in wide areas of the plasma edge.

\subsection{Experimental results}

The results of the numerical simulations can be compared with experimental results. Experimentally, we should distinguish between the detection of fast runaways (via synchrotron radiation emission) and relatively slow runaways (via electron cyclotron radiation (ECE) emission).

ECE and synchrotron radiation are both EM radiation and stem from the accelerated motion of electrons around the magnetic field lines. At low energies, the emission is a line radiation with the frequency corresponding to the electron cyclotron frequency in the microwave spectral region. At higher electron energy, radiation is emitted from (few) harmonics of the cyclotron frequency as well. The emission is predominantly perpendicular to the instantaneous direction of the magnetic field lines; in typical fusion plasmas, the radiation is optically thick-i.e. emission and re-absorption are in equilibrium; therefore the ECE is often used for the electron temperature measurement of the plasma.

It is an experimental observation that the ECE signal is a factor of four to ten times enhanced by the presence of low energy runaway electrons. The radiation is emitted perpendicular to the magnetic field lines and therefore cannot stem from highly relativistic particles. Even though it would be very intriguing to obtain information, e.g. of the distribution function of the runaways from the enhanced ECE signal, we did not succeed because of the problem of the optical thickness due to the re-absorption of the radiation. 


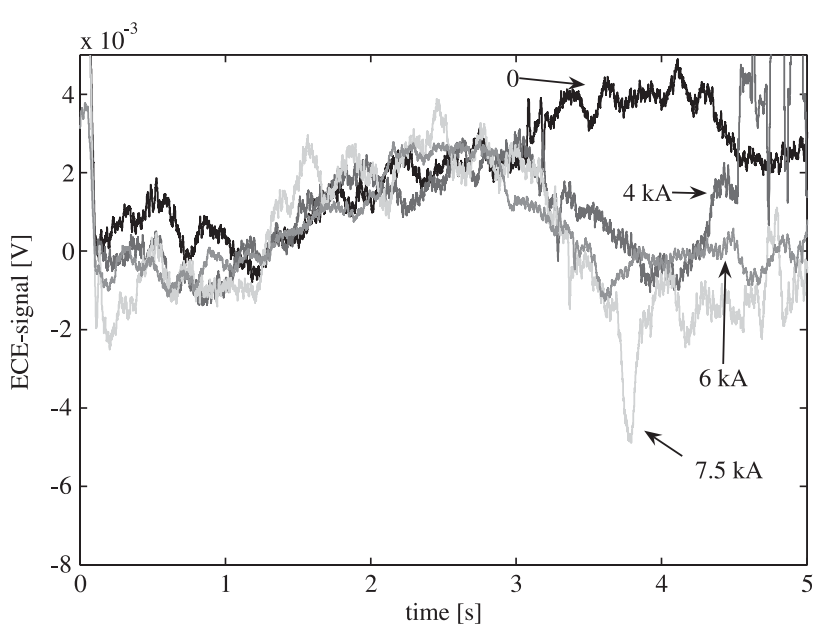

Figure 7. Experimental microwave signal, emitted by low-energetic runaway electrons as a function of time in the $6 / 2$ base mode for different perturbation currents. In order to protect the very sensitive detectors, the ECE diodes are protected by a shutter during runaway operation. For this reason, the signal is rather noisy and shows a negative offset.

With increasing electron energy, the number of harmonics increases and leads to a continuum emission when electrons are in the relativistic energy range. The Lorentz transformation forces the emission in a forward direction of the instantaneous velocity; the opening angle of the emission cone is of the order of the relativistic mass factor $\gamma$. In a tokamak the emission is in the direction of the magnetic field linesin contrast to the 'classical' emission from the synchrotron and from astrophysics objects-and it obeys the Schwinger equation $[30,65]$ : The radiation spectrum depends only on the runaway number, their energy and on the instantaneous radius of curvature; at an energy of $\epsilon=30 \mathrm{MeV}$, the emission maximum is in the IR range at about $5 \mu \mathrm{m}$. At the emission maximum, the emitted power increases with the 7th power of the energy divided by the 3rd power of the radius of curvature of the orbit [66].

Measurements of synchrotron radiation, emitted by the very high-energetic (about $30 \mathrm{MeV}$ ) runaway electrons, and microwave radiation, emitted by the lower-energetic (up to a few $\mathrm{MeV}$ ) runaway electrons, were performed at TEXTOR for the $6 / 2$ base mode operation.

Figure 7 shows the time evolution of the microwave signal, created by electrons up to $5 \mathrm{MeV}$, for different perturbation currents. The DED current is switched on at $t=3 \mathrm{~s}$, ramped to its maximum over $0.5 \mathrm{~s}$, and shut down at $t=4.5 \mathrm{~s}$. For all perturbation currents $I_{0}>0$ the microwave signal deceases more or less in the same way, especially when compared to the reference shot without a DED perturbation current $\left(I_{0}=0\right)$. This supports the theoretical result that the lower-energetic runaway electrons are heavily lost to the wall in the $6 / 2$ base mode. The dependence on the perturbation current is weak. Figure 8 shows the synchrotron signal versus time for the same shots as in figure 7 , again in $6 / 2$ base mode operation. As expected from the theoretical simulations, the amounts of highenergetic runaway losses depend strongly on the perturbation current. For $I_{0}=4 \mathrm{kA}$ only very few high-energetic runaway electrons are lost at the very end of the shot. High-energetic runaways are affected only for large perturbation currents when

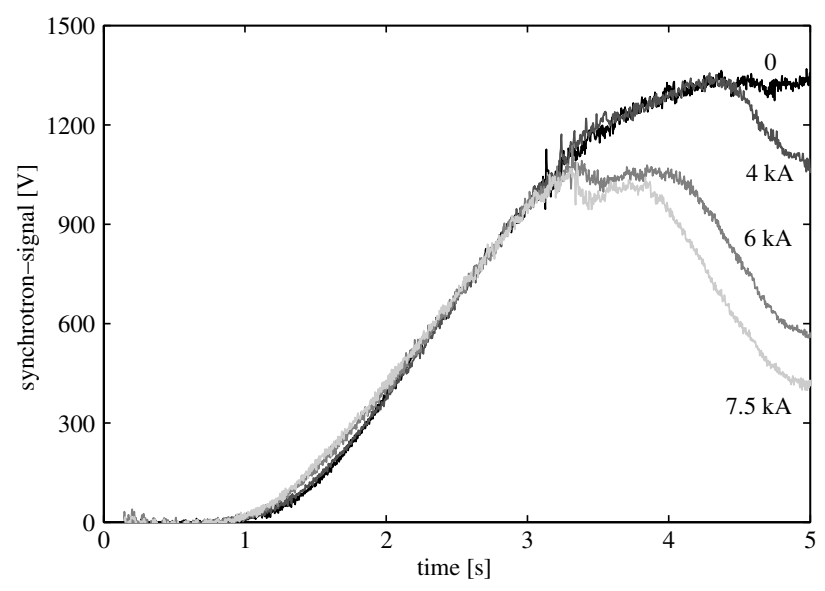

Figure 8. Experimental synchrotron radiation signal, emitted by high-energetic runaway electrons, as a function of time in the $6 / 2$ base mode, for different perturbation currents.

the threshold of the decay parameter comes into the region of high energies (see figures 5 and 6). This underlines the result that runaway electrons of about $25-30 \mathrm{MeV}$ are mostly confined inside the plasma. Remember, the energy threshold for the confinement of the ergodic zone is $7.13 \mathrm{MeV}$ for $I_{0}=$ $5 \mathrm{kA}$. By increasing the perturbation current, the loss of highenergetic runaways increases strongly. The latter is expected since the confinement threshold depends quadratically on the perturbation current. Thus, at least qualitatively, the recent measurements at TEXTOR confirm our theoretical predictions.

The interpretation of the synchrotron radiation with respect to runaway number and radial distribution is straightforward as long as the maximum energy and the radius of curvature remain constant, as is normally the case. Sudden variations of the curvature have been observed e.g. in pitch angle scattering events and they result in a sudden increase in IR-intensity. Slow variations with time scales of seconds bear some uncertainty for the interpretation because the acceleration in the loop voltage of a tokamak discharge from $1 \mathrm{MeV}$ runaway to $30 \mathrm{MeV}$ runaways requires about one second. Therefore we hesitate to interpret here the time after the switch off of the DED.

\section{Discussion and conclusion}

Starting with the general relativistic form of the Hamiltonian for a charged particle in an EM field, we applied a guidingcentre transformation to eliminate the fast gyration of the particles around the field lines. In section 2.3 the Hamiltonian and the equations of motion for the guiding-centre drift were derived in toroidal geometry in a form being appropriate for discrete mapping. For quantitative results, a model for the toroidal main field, including toroidal corrections like the Shafranov shift, was introduced in section 3.2. Combined with the perturbation field, a four-dimensional mapping was derived for the drift of relativistic particles in a (generally time-dependent) perturbation field. For static perturbations, the four-dimensional mapping reduces to a two-dimensional one.

Starting with section 3.3, the drift effects of high-energetic relativistic electrons were analysed. The shifts of the drift 
surfaces with a constant safety factor were demonstrated in figure 1. The surfaces of runaway electrons are shifted outwards with increasing kinetic energy, while the surfaces of co-passing electrons are shifted inwards. Including the perturbation, the reduction of the chaotic plasma edge with increasing kinetic particle energy was shown for electron runaways in section 4.1. Particles with high kinetic energy do not really feel the chaotic magnetic field if the latter does not propagate deep into the plasma. Low-energetic particles mainly follow the field lines. The very high-energetic electrons with kinetic energies larger than approx. $8 \mathrm{MeV}$ are totally confined inside the plasma for the $m / n=12 / 4$ base mode. The last intact drift surface is located directly beneath the wall. In the other base mode operations the perturbation penetrates deeper inside the plasma and the particles still feel the chaotic field at even higher kinetic energies. The mapping results were quantitatively confirmed by the analysis of the dependence of the escape rates on the kinetic energy in section 4.2. It was shown that the characteristic parameter $\lambda$ for the exponential decay decreases linearly with increasing kinetic energy. The critical energy level for the confinement of the ergodic zone was extrapolated for the $12 / 4,6 / 2$ and $3 / 1$ base modes.

The predictions were compared with recent measurements of runaways in TEXTOR. The experiment confirms the theoretical predictions and underlines the correctness of the estimated energy thresholds.

In appendix B we present, for completeness, the nonrelativistic limit. It should be emphasized that the highenergy effects cannot be seen in the non-relativistic case [57]. Furthermore, only drift effects of ions can be studied with the non-relativistic model. At non-relativistic energies, electrons do not show any relevant drift effects.

\section{Acknowledgments}

The work was performed under the auspices of the SFB 591 as part of the PhD thesis of Andreas Wingen. Discussions with Marcin Jakubowski, Wolfgang Laedke, Marcus Neuer and Reinhard Schlickeiser are gratefully acknowledged.

\section{Appendix A. Co-passing electrons}

For co-passing electrons the drift map also becomes more and more regular with increasing particle energy. This is similar to the findings of section 3.2. In the following we discuss the results for the $m / n=12 / 4$ base mode parameters.

Although the structures become more regular with increasing kinetic energy, the finger-structures become more distinctive and much more concentrated at the angle position of the DED coils, $\theta=\pi$. Due to the drift effects, which cause a shift of the drift surfaces towards the DED coils, as shown in figure 1 , the occurrence of the fingers around $\theta=\pi$ is expected. But the co-passing electrons are shifted into areas of stronger perturbations. One would expect similar or even stronger chaotic behaviour. On the contrary, the co-passing electrons also behave more regularly for higher energies, as can be seen in figure 9. This can only be an effect of the high kinetic energy of the electrons. Their motion inside the torus is so fast that they cannot really feel the chaotic magnetic field. Some effects of the perturbation are still present, according to

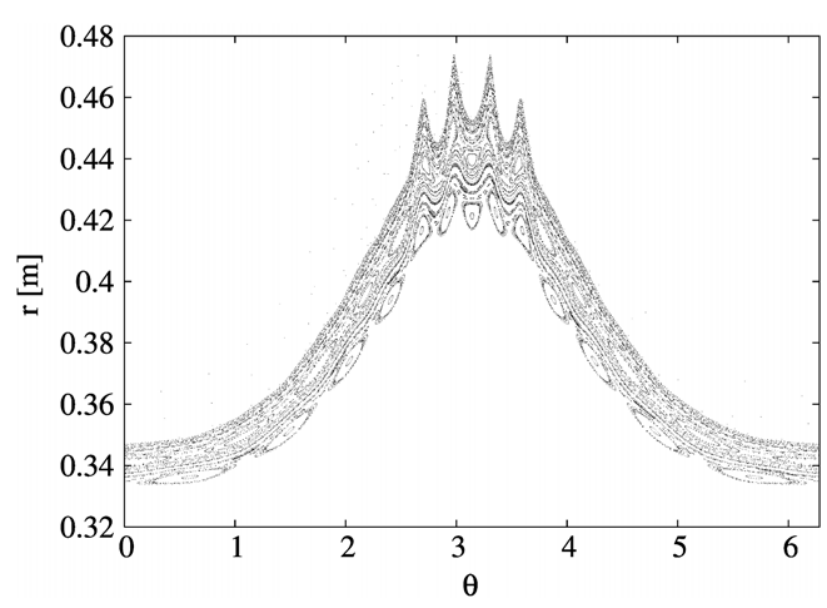

Figure 9. Drift map for $15 \mathrm{MeV}$ co-passing electrons in the $12 / 4$ base mode with $I_{0}=10 \mathrm{kA}$.

figure 9, where we can clearly see four fingers at the position of the coils. But also, as for runaway electrons, intact drift surfaces are connected to the wall, now at the inner side of the torus.

\section{Appendix B. Non-relativistic limit}

For temperatures $T$ with $k T=10-20 \mathrm{keV}$ only a few particles have energies larger than the rest energy. Most of the particles can be described non-relativistically. From our general formulation we now obtain for energies $E \ll \varepsilon_{0}$ the non-relativistic limit.

Starting from equation (32), the term

$$
\varepsilon_{0}\left(\gamma^{2}-1\right)=\varepsilon_{0}\left(\frac{-p_{t}-\phi}{\varepsilon_{0}}\right)^{2}-\varepsilon_{0}
$$

is the only part of the Hamiltonian $K$, which depends on the normalized total particle energy $\tilde{H}=-p_{t}$. By subtracting the rest energy

$$
\tilde{H}_{\mathrm{nr}}=\tilde{H}-\varepsilon_{0}=-p_{t}-\varepsilon_{0}=:-h
$$

follows. Here, we have introduced the canonical momentum $h$. It corresponds to the negative non-relativistic total energy. Inserting $h$ into equation (B.1), we obtain

$\varepsilon_{0}\left(\frac{-p_{\mathrm{t}}-\phi}{\varepsilon_{0}}\right)^{2}-\varepsilon_{0}=\varepsilon_{0}\left[\left(1+\frac{-h-\phi}{\varepsilon_{0}}\right)^{2}-1\right]$.

Going to the non-relativistic limit, we introduce the smallness parameter

$$
\mu:=\frac{-h-\phi}{\varepsilon_{0}} \ll 1 .
$$

Now we can expand the right-hand side of equation (B.3) into a power series with respect to $\mu$,

$$
\varepsilon_{0}\left[(1+\mu)^{2}-1\right] \approx 0+2 \varepsilon_{0} \mu+O\left(\mu^{2}\right)=2(-h-\phi),
$$

and neglect all higher-order terms in $\mu$. Inserting the result into the relativistic Hamiltonian $K$, we get the non-relativistic Hamiltonian

$$
K_{\mathrm{nr}}=-f_{\varphi}-\sigma\left(1+x_{c}\right) \sqrt{2\left(-h-\phi-\omega_{x} I_{x}\right)}
$$


and the non-relativistic equations of motion

$$
\begin{aligned}
\frac{\mathrm{d} z}{\mathrm{~d} \varphi} & =\frac{1}{Z_{q}}\left(1+x_{c}\right)\left[\frac{\partial f_{\varphi}}{\partial x_{c}},\right. \\
& \left.+\sigma \frac{2(-h-\phi)-\omega_{x} I_{x}-\left(1+x_{c}\right) \frac{\partial \phi}{\partial x_{c}}}{\sqrt{2\left(-h-\omega_{x} I_{x}-\phi\right)}}\right], \\
\frac{\mathrm{d} p_{z}}{\mathrm{~d} \varphi} & =\frac{\partial f_{\varphi}}{\partial z}-\sigma \frac{\partial \phi}{\partial z} \frac{1+x_{c}}{\sqrt{2\left(-h-\omega_{x} I_{x}-\phi\right)}}, \\
\frac{\mathrm{d} t}{\mathrm{~d} \varphi} & =\sigma \frac{1+x_{c}}{\sqrt{2\left(-h-\omega_{x} I_{x}-\phi\right)}}, \\
\frac{\mathrm{d} h}{\mathrm{~d} \varphi} & =\frac{\partial f_{\varphi}}{\partial t}-\sigma \frac{\partial \phi}{\partial t} \frac{1+x_{c}}{\sqrt{2\left(-h-\omega_{x} I_{x}-\phi\right)}}, \\
\frac{\mathrm{d} \vartheta_{x}}{\mathrm{~d} \varphi} & =\sigma\left(2\left(-h-\omega_{x} I_{x}-\phi\right)\right)^{-1 / 2}, \\
\frac{\mathrm{d} I_{x}}{\mathrm{~d} \varphi} & =0 .
\end{aligned}
$$

These equations agree with previous findings [57].

\section{References}

[1] Braginskii S.I. 1965 Reviews of Plasma Physics vol 1 ed M.A. Leontovich (New York: Consultants Bureau) p 205

[2] Balescu R. 1988 Transport processes in plasmas: 2. Neoclassical Transport Theory (Amsterdam: North-Holland)

[3] Hazeltine R.D. and Meiss J.D. 1992 Plasma Confinement (Redwood City, CA: Addison-Wesley)

[4] Balescu R. 2005 Aspects of Anomalous Transport in Plasmas (Bristol: Institute of Physics Publishing)

[5] Bussac M.N. 1992 Stochastic magnetic field and enhanced transport Phys. Lett. A 161365

[6] Evans T.E. et al 2004 Suppression of large edge-localized modes in high-confinement DIII-D plasmas with a stochastic magnetic boundary Phys. Rev. Lett. 92235003

[7] Finken K.H. et al 2005 Toroidal plasma rotation induced by the dynamic ergodic divertor in the TEXTOR tokamak Phys. Rev. Lett. 94015003

[8] Jakubowski M.W. et al and the TEXTOR team 2006 Effect of the change in magnetic-field topology due to an ergodic divertor on the plasma structure and transport Phys. Rev. Lett. 96035004

[9] Balescu R. 1977 Matter out of Equilibrium (London: Imperial College Press)

[10] Balescu R., Vlad M. and Spineanu F. 1998 Tokamap: a Hamiltonian twist map for magnetic field lines in a toroidal geometry Phys. Rev. E 58 951-64

[11] Balescu R. 1998 Hamiltonian nontwist map for magnetic field lines with locally reversed shear in toroidal geometry Phys. Rev. E 583781

[12] Abdullaev S.S., Finken K.H., Kaleck A. and Spatschek K.H. 1998 Twist mapping for the dynamics of magnetic field lines in a tokamak ergodic divertor Phys. Plasmas 5 196-210

[13] Abdullaev S.S., Finken K.H. and Spatschek K.H. 1999 Asymptotical and mapping methods in study of ergodic divertor magnetic field in a toroidal system Phys. Plasmas 6153

[14] Finken K.H., Abdullaev S.S., Kaleck A. and Wolf G.H. 1999 Operational space of the dynamic ergodic divertor for the TEXTOR-94 Nucl. Fusion 39637

[15] Abdullaev S.S. 2004 On mapping models of field lines in a stochastic magnetic field Nucl. Fusion 44 S12-27

[16] Finken K.H., Abdullaev S.S., Jakubowski M., Lehnen M., Nicolai A. and Spatschek K.H. 2005 The Structure of
Magnetic Field in the TEXTOR-DED Energy Technology

vol 45 (Jülich: Forschungszentrum Jülich)

http://www.fz-juelich.de/zb/ online_publikationen

[17] Abdullaev S.S. 2006 Construction of Mappings for Hamiltonian Systems and Their Applications (Berlin: Springer)

[18] Wesson J. 2004 Tokamaks 3rd edn (Oxford: Clarendon Press)

[19] Boozer A.H. 1982 Particle diffusion in tokamaks with partially destroyed magnetic surfaces Phys. Rev. Lett. 49786

[20] White R.B. and Wu Y. 1993 Plasma Phys. Control. Fusion 35 595-9

[21] Mynick H.E. 1993 Transport of energetic ions by low- $n$ magnetic perturbations Phys. Fluids B 5 1471-81

[22] Mynick H.E. 1993 Stochastic transport of MeV ions by low- $n$ magnetic perturbations Phys. Fluids B 5 2460-7

[23] DeRover M., Lopes Cardozo N.J. and Montvai A. 1996 Hamiltonian description of the topology of drift orbits of relativistic particles in a tokamak Phys. Plasmas 3 4468-77

[24] DeRover M., Lopes Cardozo N.J. and Montvai A. 1996 Motion of relativistic particles in axially symmetric and perturbed magnetic fields in a tokamak Phys. Plasmas 3 4478-88

[25] White R.B. 1998 Chaos in trapped particle orbits Phys. Rev. E 58 1774-9

[26] DeRover M., Schilham A.M., Montvai A. and Lopes Cardozo N.J. 1999 Test particle transport in perturbed magnetic fields in tokamaks Phys. Plasmas 6 2443-51

[27] Finken K.H. and Wolf G.H. 1997 Background, motivation, concept and scientific aims for building a dynamic ergodic divertor Fusion Eng. Des. 37337

[28] Knoepfel H. and Spong D.A. 1979 Nucl. Fusion 19785

[29] Gill R.D., Alper B., Edwards A.W., Ingesson L.C., Johnson M.F. and Ward D.J. 2000 Direct observations of runaway electrons during disruptions in the JET tokamak Nucl. Fusion 40 163-74

[30] Finken K.H., Watkins J.G., Rusbüldt D., Corbett W.J., Dippel K.H., Goebel D.M. and Moyer R.A. 1990 Observation of infrared sychrotron radiation from tokamak runaway electrons in TEXTOR Nucl. Fusion 30859

[31] Jaspers R., Finken K.H., Mank G., Hoenen F., Boedo J., Lopes Cardozo N.J. and Schüller F.C. 1993 Experimental investigation of runaway electron generation in TEXTOR Nucl. Fusion 331775

[32] Jaspers R., Lopes Cardozo N.J., Finken K.H., Schokker B.C., Fuchs G., Mank G. and Schüller F.C. 1994 Island of runaway electrons in the TEXTOR tokamak and relation to transport in a stochastic field Phys. Rev. Lett 724093

[33] Jaspers R., Grewe T., Finken K.H., Krämer-Flecken A., Lopes Cardozo N.J., Mank G. and Waidmann G. 1995 Observations of infrared radiation during disruptions in TEXTOR: heat pulses and runaway electrons $\mathrm{J}$. Nucl. Mater. 220-222 682

[34] Jaspers R., Lopes Cardozo N.J., Schüller F.C., Finken K.H., Grewe T. and Mank G. 1996 Disruption generated runaway electrons in TEXTOR and ITER Nucl. Fusion 36 367-73

[35] Pankratov I.M., Jaspers R., Finken K.H., Entrop I. and Mank G. 1998 Control of runaway electron secondary generation by changing $Z_{\text {eff }}$ Nucl. Fusion 38279

[36] Entrop I., Jaspers R., Lopes Cardozo N.J. and Finken K.H. 1999 Runaway snakes in TEXTOR-94 Plasma Phys. Control. Fusion 41377

[37] Finken K.H., Abdullaev S.S., Jakubowski M., Jaspers R., Lehnen M. and Zimmermann O. 2006 Losses of runaway electrons during ergodization Nucl. Fusion 46 S139-44

[38] Rosenbluth M.N. and Putvinski S.V. 1997 Theory for avalanche of runaway electrons in tokamaks Nucl. Fusion 37 1355-62

[39] Plyusnin V.V., Riccardo V., Jaspers R., Alper B., Kiptily V.G., Mlynar J., Popovichev S., de La Luna E., Andersson F. and JET EFDA contributors 2006 Study of runaway electron generation during major disruptions in JET $2006 \mathrm{Nucl}$. Fusion 46 277-84 
[40] Russo A.J. 1991 Effect of ripple on runaway electrons in tokamaks Nucl. Fusion 31117

[41] Russo A.J. and Campbell R.B. 1993 A model for disruption generated runaway electrons Nucl. Fusion 331305

[42] Kawano Y. et al 1997 Proc. 16th Int. Conf. on Fusion Energy (Montreal, Canada, 1996) vol 1 (Vienna: IAEA) p 345

[43] Kawano Y. et al 1997 Proc. 24th Eur. Conf. on Controlled Fusion and Plasma Physics (Berchtesgaden, 1997) vol 21A (Geneva: European Physical Society) p 501

[44] Ghendrih Ph, Grossman A. and Capes H. 1996 Theoretical and experimental investigations of stochastic boundaries in tokamaks Plasma Phys. Control. Fusion 381653 -724

[45] Catto P.J., Myra J.R., Wang P.W., Wootton A.J. and Bengtson R.D. 1991 Estimating the runaway diffusion coefficient in the TEXT tokamak from shift and externally applied resonant magnetic-field experiments Phys. Fluids B $32038-49$

[46] Myra J.R., Catto P.J., Wootton A.J., Bengtson R.D. and Wang P.W. Runaway electrons as a diagnostic of magnetic fluctuations in the edge plasma of the Texas Experimental Tokamak Phys. Fluids B 4 2092-7

[47] Myra J.R., Catto P.J., Mynick H.E. and Duvall R.E. 1993 Quasilinear diffusion in stochastic magnetic fields: Reconciliation of drift-orbit modification calculations Phys. Fluids B 5 1160-3

[48] Catto P.J., Myra J.R. and Wootton A.J. 1994 Analytic model for the runaway distribution function in the presence of spatial diffusion Phys. Plasmas 1 684-90

[49] Kawashima H., Nagashima K., Tamai H., Miura Y., Shoji T., Fujita T., Mori M., Sakurai S., Uesugi Y. and Takamura S. 1994 Study of runaway electron transport in edge stochastic magnetic field in the JFT-2M tokamak J. Plasma Fusion Res. 70 868-76

[50] Entrop I., Lopes Cardozo N.J., Jaspers R. and Finken K.H. 1998 Diffusion of runaway electrons in TEXTOR-94 Plasma Phys. Control. Fusion 401513

[51] Tokuda S. and Yoshino R. 1999 Simulation study on collisionless loss of runaway electrons by magnetic perturbations in a tokamak Nucl. Fusion 39 1123-32

[52] Martín-Solís J.R., Sánchez R. and Esposito B. 1999 Effect of magnetic and electrostatic fluctuations on the runaway electron dynamics in tokamak plasmas Phys. Plasmas 6 3925-33
[53] Yoshino R. and Tokuda S. 2000 Runaway electrons in magnetic turbulence and runaway current termination in tokamak discharge Nucl. Fusion 40 1293-309

[54] Helander P., Eriksson L.-G. and Andersson F. 2000 Supperssion of runaway electron avalanches by radial diffusion Phys. Plasmas 7 4106-111

[55] Harvey R.W., Chan V.S., Chiu S.C., Evans T.E., Rosenbluth M.N. and Whyte D.G. 2000 Runaway electron production in DIII-D killer pellet experiments, calculated with the CQL3D/KPRAD model Phys. Plasmas 7 4590-9

[56] Abdullaev S.S. and Finken K.H. 2002 Hamiltonian guiding center equations in a toroidal system Phys. Plasmas 9 4193-204

[57] Abdullaev S.S., Wingen A. and Spatschek K.H. 2006 Mapping of drift surfaces in toroidal systems with chaotic magnetic fields Phys. Plasmas 13042509

[58] Abdullaev S.S. 1999 A new integration method of Hamiltonian systems by symplectic maps J. Phys. A: Math. Gen. 32 2745-66

[59] Abdullaev S.S. 2002 The Hamilton-Jacobi method and Hamiltonian maps J. Phys. A: Math. Gen. 35 2811-32

[60] Kasilov S.V., Kernbichler W., Nemov V.V. and Heyn M.F. 2002 Mapping techniques for stellarators Phys. Plasmas 9 3508-25

[61] Littlejohn R.G. 1985 Differential forms and canonical variables for drift motion in toroidal geometry Phys. Fluids 282015

[62] Catto P.J., Myra J.R. and Taylor J.B. 1992 Curvature drift modifications of magnetic field maps for runaway electrons Plasma Phys. Control. Fusion 34 387-404

[63] Nguyen F., Ghendrih Ph. and Samain A. 1995 Calculation of magnetic field topology of ergodized zone in real tokamak geometry. Application to the tokamak TORE-SUPRA through the MASTOC code Technical Report DFRC/CAD Preprint EUR-CEA-FC-1539, CEA, Cadarache

[64] Myra J.R. and Catto P.J. 1992 Effects of drifts on the diffusion of runaway electrons in tokamak stochastic magnetic fields Phys. Fluids B 4 176-86

[65] Schwinger J. 1949 On the classical radiation of accelerated electrons Phys. Rev. 75 1912-25

[66] Jaspers R., Lopes Cardozo N.J., Donné A.J.H. Widderhoven H.L.M. and Finken K.H. 2001 A synchrotron radiation diagnostic to observe relativistic runaway electrons in a tokamak Rev. Sci. Instrum. 72466 